\title{
Convexity meets replication: hedging of swap derivatives and annuity options
}

\author{
Wendong Zheng and Yue Kuen Kwok* \\ Department of Mathematics \\ Hong Kong University of Science and Technology \\ Clear Water Bay, Hong Kong
}

May 6, 2010

\begin{abstract}
Convexity correction arises when one computes the expected value of an interest rate index under a probability measure other than its own natural martingale measure. As a typical example, the natural martingale measure of the swap rate is the swap measure with annuity as the numeraire. However, the evaluation of the discounted expectation of the payoff in a constant maturity swap (CMS) derivative is performed under the forward measure corresponding to the payment date. In this paper, we propose a generalization of the static replication formula by exploring the linkage between replication, convexity correction and numeraire change. We illustrate how the static replication of a CMS caplet by a portfolio of payer swaptions is related to convexity correction associated with the bond-annuity numeraire ratio. We also demonstrate the use of the generalized static replication approach for hedging the in-arrears clean index principal swaps and annuity options.
\end{abstract}

Keywords: Convexity adjustment, static replication, constant maturity swap, clean index principal swap, annuity option.

\section{Introduction}

A constant maturity swap (CMS) is an example of a basis swap. One of the legs, known as the CMS leg, is indexed to a swap rate of fixed maturity (say, 10-year swap rate). This swap rate of fixed maturity is commonly called the CMS rate, and it is reset on each of the preset fixing dates. The other leg in the CMS can be indexed either to a floating rate (say, LIBOR) or a fixed rate. The class of CMS derivatives, like the CMS caps and floors, are derivative products whose payoff structures have dependence on the CMS rates. When we consider pricing of the CMS derivatives, it is convenient to compute the expectation of

\footnotetext{
*Correspondence author; e-mail: maykwok@ust.hk
} 
the future CMS rates under the forward measure that is associated with the payment date. However, the natural martingale measure of the CMS rate is the underlying annuity. Convexity correction arises when one computes the expected value of the CMS rate under the forward measure that differs from the natural swap measure with the underlying annuity as the numeraire. The CMS convexity correction is then the difference between the expectation of the CMS rate under the forward measure and swap measure. A good review of the mathematics of convexity correction can be found in Pelsser (2003a).

Carr and Madan (1998) propose the static replication formula which demonstrates how to replicate a European contingent claim with a twice differentiable payoff using a static replication strategy involving zero coupon bonds and vanilla call and put options. It has been well known among practitioners in the fixed income markets that a CMS caplet can be replicated by a portfolio of payer swaptions whose terminal payoff matches with that of the CMS caplet. The linkage between convexity correction and static replication of CMS derivatives has been explored in the literature (Hagan, 2003; Reiner and Selami, 2006; Mercurio and Pallavicini, 2006). In this paper, we would like to illustrate the linkage between convexity correction and static replication through pricing and hedging of various CMS products and annuity instruments, like the CMS caplets, in-arrears clean index principal swaps and annuity options. We also propose a generalized static replication approach that allows for more general choices of option-type instruments (not just limited to vanilla options as in the Carr-Madan replication formula). In addition, with regard to convenient valuation of the contingent claim, our generalized static replication formula allows for the flexibility on the choices of different numeraires for the contingent claims and the replicating instruments.

This paper is organized as follows. In the next section, we present the approximate static replication of a CMS caplet using a portfolio of payer swaptions with varying discrete strike prices. This approximate hedging procedure has been widely used by practitioners. By taking the continuous limit of the differential interval width between the strike prices of the payer swaptions, we are able to relate the continuous limit of this approximate static replication with other replication procedures that are derived using convexity correction arguments. This example of static replication of a CMS caplet serves to provide the linkage between replication and convexity correction. In Section 3, we derive the generalization of the static replication approach that incorporates change of measure into the formulation and allows for the use of a wider class of option-type products in the replicating portfolio. We then illustrate how to apply the generalized static replication approach to the pricing and hedging of the in-arrears clean index principal swaps and annuity options. In Section 4, we present sample calculations of finding the replication portfolios of CMS caplets and annuity options. Concluding remarks are presented in the last section.

\section{Static replication of a CMS caplet}

Since both the CMS caplet and the payer swaption share the CMS rate as the underlying, practitioners have commonly adopted the approximate hedging strategy of static replication of the less liquid CMS caplet using a portfolio of the more 
liquid payer swaptions with discrete strikes. In this section, the continuous limit of this approximate replication procedure is shown to agree with that derived using the convexity correction technique (Hagan, 2003; Mercurio and Pallavicini, 2006).

First, we fix the mathematical notations for the tenor structure, bond price function and swap rate. The spot time is taken to be time 0 and the tenor structure of the reference swap rate is assumed to be $\left\{T_{0}, T_{1}, \cdots, T_{n}\right\}$. Here, $T_{0}$ is the start date of the swap, $\left\{T_{i}, i=0,1, \cdots, n-1\right\}$ are the reset dates and $\left\{T_{i}, i=1, \cdots, n\right\}$ are the payment dates. We let $\delta_{i}$ be the accrual factor for the time interval $\left[T_{i-1}, T_{i}\right]$ based on certain day count convention, and write $\delta$ for all $\delta_{i}$ if a constant accrual factor is assumed. The time- $t$ price of the $T_{i^{-}}$ maturity discount bond is denoted by $B\left(t, T_{i}\right), i=0,1, \cdots, n$, where $t \in\left[0, T_{0}\right]$. The annuity stream with $k$ payments is defined by $A_{k}(t)=\sum_{i=1}^{k} \delta_{i} B\left(t, T_{i}\right)$, for $k=1,2, \cdots, n$, and $A(t)$ is used to denote $A_{n}(t)$ for short. We let $S\left(t ; T_{0}, T_{i}\right)$ denote the forward swap rate at time $t, t \in\left[0, T_{0}\right]$, with start date $T_{0}$ and payment dates $\left\{T_{1}, T_{2}, \cdots, T_{i}\right\}, i=1,2, \cdots, n$. Provided that no confusion arises, we may use $S_{t}$ to denote $S\left(t ; T_{0}, T_{n}\right)$ for short. As usual, we use $E_{T_{i}}[\cdot]$ to denote the expectation under the forward measure $\mathbb{Q}_{T_{i}}$, where the $T_{i}$-maturity discount bond $B\left(t, T_{i}\right)$ is used as the numeraire, $i=1, \cdots, n$. Also, $E_{\mathbb{Q}_{N}}[\cdot]$ denotes the expectation under the martingale measure $\mathbb{Q}_{N}$ associated with the numeraire $N(t)$.

The most basic CMS derivative is the CMS caplet whose payoff on the payment date $T_{p}$, where $T_{p} \geq T_{0}$, is given by

$$
F\left(S_{T_{0}}, T_{p} ; K\right)=\left(S_{T_{0}}-K\right)^{+} .
$$

Here, the notation $(\cdot)^{+}$denotes $\max \{\cdot, 0\}$ and $K$ is the strike of the caplet. Recall that a European swaption allows its holder to enter into a fixed-floating swap of the preset tenor and pre-specified fixed rate $K$ on maturity. A payer (receiver) swaption allows the holder to pay (receive) the fixed rate. Swaptions can be either physically settled or cash-settled. We would like to illustrate how to replicate the caplet payment on the payment date $T_{p}$ approximately using a portfolio of physically settled payer swaptions with discrete strikes $K+m \Delta x, m=0,1,2, \cdots$, where $\Delta x$ represents a small increment on the strike rate starting from $K$, then $K+\Delta x, K+2 \Delta x$, etc. Let $N_{m}$ denote the notional amount of the payer swaption with strike $K+m \Delta x, m=0,1,2, \cdots$. We illustrate how to determine $N_{0}, N_{1}, N_{2}, \cdots$ successively in order that the caplet payoff at $T_{p}$ agrees with that of the replicating portfolio of payer swaptions under various scenarios of the observed swap rate at $T_{0}$, that is, the realized value of $S_{T_{0}}$.

When $S_{T_{0}} \leq K$, the caplet has zero payoff and all the payer swaptions in the replicating portfolio are not in-the-money, so matching of the two payoffs is achieved. Next, we determine the notional amount of each of the payer swaptions successively by matching the payoffs of the caplet and replicating portfolio at various possible discrete values assumed by $S_{T_{0}}$.

First, suppose $S_{T_{0}}=K+\Delta x$, the caplet's payoff is $\Delta x$ at $T_{p}$ and the corresponding discounted value at $T_{0}$ is $\left.\Delta x B\left(T_{0}, T_{p}\right)\right|_{S_{T_{0}}=K+\Delta x}$. On the other hand, only the payer swaption with strike rate $K$ is in-the-money while all the other swaptions with higher strike rate have zero payoff. The time- $T_{0}$ payoff of the payer swaption with strike rate $K$ when $S_{T_{0}}=K+\Delta x$ is given in the form of 
an annuity $\left.\Delta x \sum_{i=1}^{n} \delta_{i} B\left(T_{0}, T_{i}\right)\right|_{S_{T_{0}}=K+\Delta x}$. To achieve matching of the payoffs of the caplet and replicating portfolio when $S_{T_{0}}=K+\Delta x$, the notional amount $N_{0}$ must be set uniquely equal to the following bond-annuity ratio:

$$
N_{0}=\left.\frac{B\left(T_{0}, T_{p}\right)}{\sum_{i=1}^{n} \delta_{i} B\left(T_{0}, T_{i}\right)}\right|_{S_{T_{0}}=K+\Delta x} .
$$

Naturally, the above bond-annuity ratio exhibits dependence on the swap rate $S_{T_{0}}$. Similar to Hagan (2003), we write formally the functional dependence of the bond-annuity ratio on $S_{T_{0}}$ in the form

$$
G\left(S_{T_{0}}\right)=\frac{B\left(T_{0}, T_{p}\right)}{\sum_{i=1}^{n} \delta_{i} B\left(T_{0}, T_{i}\right)}
$$

Accordingly, we may express the notional of the payer swaption with strike rate $K$ in terms of $G$ as follows:

$$
N_{0}=G(K+\Delta x) .
$$

Next, we determine the notional amount $N_{1}$ of the payer swaption with strike $K+\Delta x$ by matching the payoffs when $S_{T_{0}}$ assumes the value $K+2 \Delta x$. Under such scenario, only the two swaptions with respective strike $K$ and $K+\Delta x$ are in-the-money so that

$$
\left.\left(2 N_{0}+N_{1}\right) \Delta x \sum_{i=1}^{n} \delta_{i} B\left(T_{0}, T_{i}\right)\right|_{S_{T_{0}}=K+2 \Delta x}=\left.2 \Delta x B\left(T_{0}, T_{p}\right)\right|_{S_{T_{0}}=K+2 \Delta x}
$$

giving

$$
N_{1}=2[G(K+2 \Delta x)-G(K+\Delta x)] .
$$

In general, by matching the payoffs of the caplet and the replicating portfolio when $S_{T_{0}}=K+(m+1) \Delta x$, the notional amount $N_{m}, m \geq 1$, must be set uniquely equal to

$$
\begin{aligned}
N_{m}= & (m+1)[G(K+(m+1) \Delta x)-G(K+m \Delta x)] \\
& -(m-1)[G(K+m \Delta x)-G(K+(m-1) \Delta x)] .
\end{aligned}
$$

Let $C_{0}(K)$ denote the time- 0 value of the payer swaption with strike rate $K$ and $V_{0}^{\text {caplet }}$ denote the time- 0 value of the CMS caplet. Since the payoff of the replicating portfolio agrees with that of the caplet at discrete strikes according to this approximate static replication procedure, by applying the no-arbitrage principle, the fair value of the CMS caplet is approximately given by the value of this portfolio of payer swaptions with discrete strikes:

$$
V_{0}^{\text {caplet }} \approx N_{0} C_{0}(K)+\sum_{m=1}^{\infty} N_{m} C_{0}(K+m \Delta x) .
$$

There exists a slight mismatch of the two payoffs if $S_{T_{0}}$ does not fall exactly on one of these discrete strike values. The order of approximation can be shown to be $O\left(\Delta x^{2}\right)$. In reality, the replicating portfolio may include payer swaptions with strike up to $K+M \Delta x$ for some sufficiently large positive integer value $M$. The value of the payer swaption with exceedingly large value of strike would be 
vanishingly small, so the error incurred by this truncation to a finite portfolio of sawptions would be small.

\section{Continuous limit}

Apparently, the replication using payer swaptions with discrete strikes corresponds to the discretization in the space of the strike price of the interval $[K, \infty)$ into discrete sub-intervals of uniform width $\Delta x$. It would be interesting to consider the continuous limit of the approximation formula (2.6) when $\Delta x \rightarrow 0$. Writing $x_{m}=K+m \Delta x$ for notational convenience, and assuming $G$ to be twice differentiable, we obtain

$$
\begin{aligned}
N_{m} & =2 G^{\prime}\left(x_{m}\right) \Delta x+G^{\prime \prime}\left(x_{m}\right) m \Delta x^{2}+O\left(\Delta x^{3}\right) \\
& =2 G^{\prime}\left(x_{m}\right) \Delta x+G^{\prime \prime}\left(x_{m}\right)\left(x_{m}-K\right) \Delta x+O\left(\Delta x^{3}\right) .
\end{aligned}
$$

In the continuous limit $\Delta x \rightarrow 0$, the price formula of the CMS caplet then becomes

$$
V_{0}^{\text {caplet }}=G(K) C_{0}(K)+\int_{K}^{\infty}\left[2 G^{\prime}(x)+G^{\prime \prime}(x)(x-K)\right] C_{0}(x) d x .
$$

The above caplet price formula is essentially the same as that derived independently by Hagan (2003) and Mercurio and Pallavicini (2006) using convexity correction arguments. Indeed, Hagan obtains the caplet price formula in the form:

$$
\begin{aligned}
V_{0}^{\text {caplet }}= & \frac{B\left(0, T_{p}\right)}{A(0)} C_{0}(K)+\left[G(K)-\frac{B\left(0, T_{p}\right)}{A(0)}\right] C_{0}(K) \\
& +\int_{K}^{\infty}\left[2 G^{\prime}(x)+G^{\prime \prime}(x)(x-K)\right] C_{0}(x) d x .
\end{aligned}
$$

The sum of the last two terms is considered by Hagan to be the convexity correction for the CMS caplet. Thus, we observe the analogy between replication and convexity correction. In particular, when the caplet is at-the-money so that the strike observes the following relation:

$$
G(K)=G\left(S_{0}\right)=\frac{B\left(0, T_{p}\right)}{A(0)}
$$

then the convexity correction is merely given by the integral term in Eq. (2.8).

\section{Generalized static replication formula}

Let $\left\{X_{t}\right\}$ denote the underlying asset price process and $f\left(X_{T}\right)$ denote the time- $T$ payoff of a European contingent claim, where $f$ is a function that is at least twice differentiable. Carr and Madan (1998) show that

$$
\begin{aligned}
f\left(X_{T}\right)= & f(\kappa)+f^{\prime}(\kappa)\left[\left(X_{T}-\kappa\right)^{+}-\left(\kappa-X_{T}\right)^{+}\right] \\
& +\int_{0}^{\kappa} f^{\prime \prime}(x)\left(x-X_{T}\right)^{+} d x+\int_{\kappa}^{\infty} f^{\prime \prime}(x)\left(X_{T}-x\right)^{+} d x,
\end{aligned}
$$

where $\kappa \geq 0$ is an arbitrary real number. The above static replication formula reveals the static replication of a European contingent claim using zero-coupon bonds and a portfolio of vanilla call and put options. However, it is desirable to extend the static replication procedure so as to cope with the following generalizations: 
1. The contingent claims and the replicating instruments may use different choices of numeraires that are adopted for convenient valuation.

2. In order to allow for greater flexibility in the choices of liquid replicating instruments, the payoff structure of the option-type replicating instruments may not resemble exactly the vanilla call or put option payoff.

For example, in the static replication of the CMS caplet by a portfolio of payer swaptions, the payoff of the CMS caplet with strike $x$ when normalized by the numeraire $B\left(T_{0}, T_{p}\right)$ is $\left(S_{T_{0}}-x\right)^{+}$while the payoff of the physically settled payer swaption with strike $x$ when normalized by the numeraire $A\left(T_{0}\right)$ is $\left(S_{T_{0}}-x\right)^{+}$. On the other hand, suppose we use the cash-settled payer swaptions as the replicating instruments, the payoff of the cash-settled payer swaption with strike $x$ when normalized by the numeraire $B\left(t, T_{0}\right)$ becomes the non-vanilla style option payoff $\sum_{i=1}^{n} \frac{\delta}{\left(1+\delta S_{T_{0}}\right)^{i}}\left(S_{T_{0}}-x\right)^{+}$, where a constant accrual factor $\delta$ is assumed.

In this section, we would like to derive a generalized replication formula that allows the use of tradeable instruments with more generalized form of optiontype payoffs in the replication of a European contingent claim. Our generalized replication formula also allows the contingent claim and replicating instruments to use different choices of numeraires that are preferred for convenient valuation.

Let $f\left(X_{T}\right)$ denote the time- $T$ payoff of a European contingent claim relative to the numeraire $M(T)$, where $f$ is twice differentiable and $X_{t}$ is the underlying asset price process. The time- $T$ payoff function of the call-type and put-type replicating instruments with strike $x$ relative to the numeraire $N(T)$ take the form $g\left(X_{T}\right)\left(X_{T}-x\right)^{+}$and $g\left(X_{T}\right)\left(x-X_{T}\right)^{+}$, respectively, where $g$ is some positive and twice differentiable function. Let the time- 0 value of these call-type and puttype instruments be denoted by $C_{0}(x)$ and $P_{0}(x)$, respectively.

Proposition 1. Suppose the numeraire ratio $M(T) / N(T)$ can be expressed as a function of $X_{T}$, i.e., $M(T) / N(T)=\Lambda\left(X_{T}\right)$, where $\lambda$ is assumed to be twice differentiable. Suppose there exists $\kappa \geq 0$ such that $f(\kappa)=0$. Let $V_{0}$ be the time-0 value of the contingent claim and let $w(x)=\frac{f(x) \Lambda(x)}{g(x)}$, then we have the following static replication formula:

$$
\begin{aligned}
V_{0}= & w^{\prime}(\kappa)\left[C_{0}(\kappa)-P_{0}(\kappa)\right] \\
& +\int_{0}^{\kappa} w^{\prime \prime}(x) P_{0}(x) d x+\int_{\kappa}^{\infty} w^{\prime \prime}(x) C_{0}(x) d x .
\end{aligned}
$$

The proof of the proposition is presented in Appendix A.

The challenge in the use of the generalized static replication formula is to find the functional dependence of the numeraire ratio in terms of the underlying asset price process $X_{T}$. Next, we would like to illustrate how to apply formula (3.2) to perform the static replication of the CMS caplets, in-arrears clean index principal swaps and annuity options.

\section{CMS caplets}

Using the generalized static replication formula (3.2), it is relatively straightforward to reproduce the replication formula (2.8) which reveals the static replication of the CMS caplet using a portfolio of payer swaptions. When normalized by 
the annuity numeraire $A\left(T_{0}\right)$, the payoff of the physically settled payer swaption is $\left(S_{T_{0}}-x\right)^{+}$, giving $g\left(S_{T_{0}}\right) \equiv 1$. The payoff of the caplet when normalized by $B\left(T_{0}, T_{p}\right)$ is $f\left(S_{T_{0}}\right)=\left(S_{T_{0}}-K\right)^{+}$. Now, the numeraire ratio is $\frac{B\left(T_{0}, T_{p}\right)}{A\left(T_{0}\right)}$ which can be assumed to be $\Lambda\left(S_{T_{0}}\right)$. After substituting the above expressions into formula (3.2), the resulting replication of the CMS caplet via a portfolio of physically settled payer swaptions agrees with that given in Eq. (2.8).

Alternatively, if the terminal payoff of the payer swaption is cash-settled, we take $N(t)$ to be the bond price $B\left(t, T_{0}\right)$ so that the time- $T_{0}$ payoff of the payer swaption is $g\left(S_{T_{0}}\right)\left(S_{T_{0}}-x\right)^{+}$, where $g\left(S_{T_{0}}\right)=\sum_{i=1}^{n} \frac{\delta}{\left(1+\delta S_{T_{0}}\right)^{i}}$. The corresponding numeraire ratio can be written as

$$
\Lambda\left(S_{T_{0}}\right)=\frac{B\left(T_{0}, T_{p}\right)}{B\left(T_{0}, T_{0}\right)} .
$$

Furthermore, we obtain

$$
w(x)=\frac{(x-K)^{+} \Lambda(x)}{\sum_{i=1}^{n} \frac{\delta}{(1+\delta x)^{i}}} .
$$

By taking $\kappa=0$ in the generalized static replication formula, the replication of the CMS caplet by a portfolio of cash-settled payer swaptions can be expressed as

$$
V_{0}^{\text {caplet }}=\int_{0}^{\infty} w^{\prime \prime}(x) C_{0}(x) d x .
$$

Since $w^{\prime \prime}(x)=0$ for $x<K$, Eq. (3.3) can be further reduced to

$$
V_{0}^{\text {caplet }}=w^{\prime}(K) C_{0}(K)+\int_{K}^{\infty} w^{\prime \prime}(x) C_{0}(x) d x
$$

When we take $T_{p}=T_{0}$, we obtain $\Lambda(x) \equiv 1$. In this case, the replication formula agrees with that given by Reiner and Sellami (2006).

As a remark, the above procedure requires the specification of the functional dependence of the numeraire ratios in terms of $S_{T_{0}}$. Though the discount factors and the swap rate are related, sometimes it may be quite tricky to find the explicit form of $\Lambda\left(S_{T_{0}}\right)$. Under certain yield curve models, Hagan (2003) and Mercurio and Pallavicini (2006) have shown that several forms of functional dependence do provide reasonably good approximation. Some of these choices of $\Lambda\left(S_{T_{0}}\right)$ are discussed in Section 4 [see Eqs. (4.1) and (4.2)].

\section{In-arrears clean index principal swaps}

The in-arrears clean index principal swap (IPS) is a variation of the standard IPS embedded with two additional features: (i) the LIBORs are reset in arrears; (ii) the notional principal $P$ is reset according to the LIBOR prevailing on the payment date. To be specific, the time- $T_{i}$ net value of the swap transaction payments to the floating rate receiver is given by $\delta_{i} P\left(L_{i}\left(T_{i}\right)\right)\left[L_{i}\left(T_{i}\right)-K\right]$. Here, the notional principal $P\left(L_{i}\left(T_{i}\right)\right)$ has dependence on the in-arrears LIBOR $L_{i}$ observed at the prevailing time $T_{i}$. The appropriate replicating instruments would be the vanilla caplets and floorlets whose terminal payoffs at time $T_{i+1}$ are

$$
\delta_{i+1}\left(L_{i}\left(T_{i}\right)-x\right)^{+} \text {and } \delta_{i+1}\left(x-L_{i}\left(T_{i}\right)\right)^{+},
$$


respectively. Now, the numeraire ratio should be

$$
\Lambda\left(L_{i}\left(T_{i}\right)\right)=\frac{B\left(T_{i}, T_{i}\right)}{B\left(T_{i}, T_{i+1}\right)}=1+\delta_{i+1} L_{i}\left(T_{i}\right)
$$

and $g\left(L_{i}\left(T_{i}\right)\right)=\delta_{i+1}$. Also, $\kappa$ should be conveniently chosen to be $K$. Substituting all of the above quantities into the generalized static replication formula, we obtain the static replication of this IPS by a portfolio of caplets and floorlets.

As an illustrative example, suppose we choose the notional $P\left(L_{i}\left(T_{i}\right)\right)$ to be $\mathbf{1}_{\{x \geq K\}}+[b(x-K)+1]^{+} \mathbf{1}_{\{x<K\}}$, where $b>0$. The notional, which is bounded below by zero, has its value decrease linearly when LIBOR falls below the strike rate $K$. This feature provides the floating rate receiver the protection of mitigating the loss by reducing the principal of the swap. By the generalized static replication formula, the net time- 0 value of the swap payment transacted at $T_{i}$ is given by

$$
\begin{aligned}
V_{0}^{i}= & \frac{\delta_{i}}{\delta_{i+1}}\left\{\left(1+\delta_{i+1} K\right)\left[C_{0}(K)-P_{0}(K)\right]-\left[1+\delta_{i+1}\left(K-\frac{1}{b}\right)\right] P_{0}\left(K-\frac{1}{b}\right) \mathbf{1}_{\left\{K \geq \frac{1}{b}\right\}}\right\} \\
& +\int_{\left(K-\frac{1}{b}\right)^{+}}^{K} 2 b \frac{\delta_{i}}{\delta_{i+1}}\left(1+\delta_{i+1} x\right) P_{0}(x) d x+\int_{0}^{K} 2 \delta_{i} P_{0}(x) d x+\int_{K}^{\infty} 2 \delta_{i} C_{0}(x) d x .
\end{aligned}
$$

Here, $C_{0}(x)$ and $P_{0}(x)$ denote the time- 0 value of the vanilla caplet and floorlet with respective terminal payoff $\delta_{i+1}\left[L_{i}\left(T_{i}\right)-x\right]^{+}$and $\delta_{i+1}\left[x-L_{i}\left(T_{i}\right)\right]^{+}$at $T_{i+1}$. If we let $b \rightarrow 0$, then the notional principal tends to the constant unit value. The in-arrears IPS reduces to an in-arrears swap, and formula (3.5) becomes

$$
\begin{aligned}
V_{0}^{i}= & \frac{\delta_{i}}{\delta_{i+1}}\left(1+\delta_{i+1} K\right)\left[C_{0}(K)-P_{0}(K)\right] \\
& +\int_{0}^{K} 2 \delta_{i} P_{0}(x) d x+\int_{K}^{\infty} 2 \delta_{i} C_{0}(x) d x
\end{aligned}
$$

which is the static replication formula for the in-arrears swaplet. On the other hand, if we let $b \rightarrow \infty$, then the payoff equals $\delta_{i}\left[L_{i}\left(T_{i}\right)-K\right]^{+}$. The in-arrears IPS swaplet becomes a caplet on the in-arrears LIBOR. In this case, the static replication formula (3.5) reduces to

$$
V_{0}^{i}=\frac{\delta_{i}}{\delta_{i+1}}\left(1+\delta_{i+1} K\right) C_{0}(K)+\int_{K}^{\infty} 2 \delta_{i} C_{0}(x) d x
$$

which is the usual convexity correction formula for the $T_{i}$-maturity caplet on in-arrears LIBOR.

\section{Annuity options}

A forward start annuity pays the amount $c_{i}$ at the future time $T_{i}, i=0,1, \cdots, n$. An option on this annuity gives the holder on the expiration date $T_{0}$ the right but not the obligation to enter into this annuity at the fixed strike $A$. The terminal payoff at time $T_{0}$ of the annuity option is given by

$$
V_{T_{0}}^{\text {a.o. }}=\max \left\{\sum_{i=0}^{n} c_{i} B\left(T_{0}, T_{i}\right)-A, 0\right\} .
$$


Pelsser (2003b) proposes a procedure of performing the static replication of the annuity option using a portfolio of receiver swaptions with different swap tenors. We would like to illustrate how to derive an alternative static replication portfolio via the generalized static replication formula (3.2).

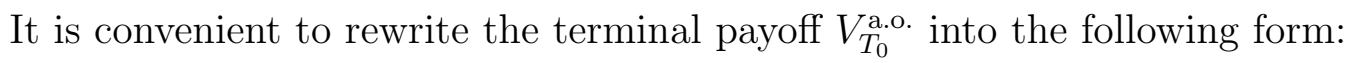

$$
A_{n}\left(T_{0}\right)\left[\sum_{i=1}^{n} \frac{c_{i} B\left(T_{0}, T_{i}\right)}{A_{n}\left(T_{0}\right)}-\frac{A-c_{0}}{A_{n}\left(T_{0}\right)}\right]^{+} .
$$

We may assume

$$
\frac{B\left(T_{0}, T_{i}\right)}{A_{n}\left(T_{0}\right)}=f_{i}\left(S_{T_{0}}\right), \quad i=0,1, \cdots, n .
$$

This is consistent with the observation that the term structures of interest rates almost move in parallel at long maturities so that the long end of the yield curve is well described by the swap rate. With the use of the numeraire $M(t)=A_{n}(t)$, the normalized terminal payoff of the annuity option can be expressed as

$$
f\left(S_{T_{0}}\right)=\frac{V_{T_{0}}^{\text {a.o. }}}{M\left(T_{0}\right)}=\left[\sum_{i=1}^{n} c_{i} f_{i}\left(S_{T_{0}}\right)-\left(A-c_{0}\right) f_{0}\left(S_{T_{0}}\right)\right]^{+} .
$$

The fixed strike $A$ should be chosen such that $A-c_{0}$ remains positive, otherwise the optionality feature in the annuity option becomes superfluous. When the physically settled swaptions are adopted as the replicating instruments, the corresponding numeraire $N(t)$ for the swaptions is the annuity $A_{n}(t)$. The numeraire ratio is then given by

$$
\frac{M(t)}{N(t)}=1,
$$

and $g\left(S_{T_{0}}\right)=1$. Our generalized formula gives the following static replication formula:

$$
V_{0}^{\text {a.o. }}=f^{\prime}(\kappa)\left[C_{0}(\kappa)-P_{0}(\kappa)\right]+\int_{0}^{\kappa} f^{\prime \prime}(x) P_{0}(x) d x+\int_{\kappa}^{\infty} f^{\prime \prime}(x) C_{0}(x) d x
$$

where $\kappa$ has been chosen such that $f(\kappa)=0, C_{0}(x)$ and $P_{0}(x)$ denote the time- 0 value of the unit notional payer swaption and receiver swaption with strike $x$, respectively.

As an illustrative example, suppose we take the annualized annuity rate $\mu$ to be constant, that is, $\mu=\frac{c_{i}}{\delta_{i}}, i=1, \cdots, n$. In this case, the terminal payoff $V_{T_{0}}^{\text {a.o. }}$ can be simplified as

$$
A_{n}\left(T_{0}\right)\left[\mu-\frac{A-c_{0}}{A_{n}\left(T_{0}\right)}\right]^{+} .
$$

Take $T_{p}=T_{0}$ in the function $G\left(S_{T_{0}}\right)$ defined earlier in Eq. (2.3), we then have

$$
G\left(S_{T_{0}}\right)=\frac{B\left(T_{0}, T_{0}\right)}{A_{n}\left(T_{0}\right)}=\frac{1}{A_{n}\left(T_{0}\right)} .
$$

We define the function $\widetilde{f}(x)=\mu-\left(A-c_{0}\right) G(x)$ so that $f(x)=[\tilde{f}(x)]^{+}$. The first-order and second-order derivatives of $f$ are found to be

$$
\begin{aligned}
f^{\prime}(x) & =\widetilde{f}^{\prime}(x) \mathbf{1}_{\{\tilde{f}(x) \geq 0\}}, \\
f^{\prime \prime}(x) & =\delta(\widetilde{f}(x)) \widetilde{f}^{\prime}(x)^{2}+\mathbf{1}_{\{\widetilde{f}(x) \geq 0\}} \widetilde{f}^{\prime \prime}(x),
\end{aligned}
$$


where $\delta(\cdot)$ is the Dirac delta function. It is observed that $G(x) \rightarrow \infty$ monotonically as $x \rightarrow \infty$, and $G(x)$ grows asymptotically like a linear function. Since $A-c_{0}$ and $\mu$ are positive, thus $\widetilde{f}(x)$ is monotonically decreasing with $\widetilde{f}(0)>0$ and $\widetilde{f}(\infty)=-\infty$. Therefore, there exists $K>0$ such that $\widetilde{f}(x) \geq 0$ if and only if $x \leq K$. Consequently, we may choose $\kappa$ to be the positive infinity so that the static replication involves the receiver swaptions only. Observing that $f^{\prime}(\kappa)=0$ when $\kappa=\infty$, we obtain the following static replication of the annuity option using a portfolio of receiver swaptions:

$$
\begin{aligned}
V_{0}^{\text {a.o. }} & =\int_{0}^{\infty} f^{\prime \prime}(x) P_{0}(x) d x \\
& =\left(A-c_{0}\right) G^{\prime}(K) P_{0}(K)-\int_{0}^{K}\left(A-c_{0}\right) G^{\prime \prime}(x) P_{0}(x) d x .
\end{aligned}
$$

\section{Numerical results}

To demonstrate the interplay between static replication and convexity correction, we performed the numerical calculations that find the corresponding replicating portfolio for the CMS caplet and the annuity option based on formulas (2.6) and (3.8). First of all, it is necessary to establish the functional dependence of the bond-annuity ratio on $S_{T_{0}}$ [see Eq. (2.3)] based on certain assumptions on the yield curve. Here, we consider two common types of yield curve models: the standard model and the parallel shifts model (Hagan, 2003). These two models are known to work well when the pricing problems are more concerned with the long-maturity segment of the yield curve. In the present context, the CMS rates are usually the medium-term to long-term rates, and the underlying annuity is also usually long-term. Therefore, the adoption of these two yield curve models is justified.

In the standard model, we assume a constant accrual factor $\frac{1}{q}$ ( $q=1$ for annual, $q=2$ for semi-annual), and the discount bond prices can be expressed in terms of $S_{T_{0}}$ as follows:

$$
B\left(T_{0}, T_{i}\right)=\frac{1}{\left(1+\frac{S_{T_{0}}}{q}\right)^{i}}, i=1,2, \cdots, n .
$$

Also, we assume

$$
B\left(T_{0}, T_{p}\right)=\frac{1}{\left(1+\frac{S_{T_{0}}}{q}\right)^{p}}
$$

so that

$$
G\left(S_{T_{0}}\right)=\frac{S_{T_{0}}}{\left(1+\frac{S_{T_{0}}}{q}\right)^{p}\left[1-\left(1+\frac{S_{T_{0}}}{q}\right)^{-n}\right]} .
$$

On the other hand, when we assume a parallel yield curve shift of a fixed amount $x$ in the parallel shifts model, the bond prices at time 0 and time $t$ are related by

$$
\frac{B\left(t, T_{i}\right)}{B\left(t, T_{0}\right)}=\frac{B\left(0, T_{i}\right)}{B\left(0, T_{0}\right)} e^{-\left(T_{i}-T_{0}\right) x}, i=1,2, \cdots, n .
$$


The parallel shift amount $x$ is implicitly determined by the following equation:

$$
S_{T_{0}}=\frac{B\left(0, T_{0}\right)-B\left(0, T_{n}\right) e^{-\left(T_{n}-T_{0}\right) x}}{\sum_{k=1}^{n} \delta_{k} B\left(0, T_{k}\right) e^{-\left(T_{k}-T_{0}\right) x}}
$$

Under the parallel shifts model, we have

$$
G\left(S_{T_{0}}\right)=\frac{S_{T_{0}} e^{-\left(T_{p}-T_{0}\right) x}}{1-\frac{B\left(0, T_{n}\right)}{B\left(0, T_{0}\right)} e^{-\left(T_{n}-T_{0}\right) x}}
$$

where $x$ has an implicit dependence on $S_{T_{0}}$.

In Figures 1(a) and 1(b), we plot the dependence of $G\left(S_{T_{0}}\right)$ on the swap rate $S_{T_{0}}$ based on the flat yield curve model and parallel shifts model, respectively, for various values of $n$ (the number of payment dates in the tenor structure $\left.\left\{T_{0}, T_{1}, \cdots, T_{n}\right\}\right)$. We assume $T_{p}=T_{1}$, and $q=2$ in our calculations. The values of the discount factors in the initial discount curve are tabulated in Table 1. The "almost linear" property of $G$ is observed in the figures even when $S_{T_{0}}$ assumes a conceivably large value; that is consistent with the use of the linear approximation assumption in the Linear Swap Rate Model (Pelsser, 2003b).

Discount Factors from $T_{0}$ to $T_{6}$

\begin{tabular}{|c|c|c|c|c|c|c|}
\hline$T_{0}$ & $T_{1}$ & $T_{2}$ & $T_{3}$ & $T_{4}$ & $T_{5}$ & $T_{6}$ \\
\hline 0.9537 & 0.9330 & 0.9139 & 0.8953 & 0.8568 & 0.8383 & 0.8204 \\
\hline
\end{tabular}

Discount Factors from $T_{7}$ to $T_{13}$

\begin{tabular}{|c|c|c|c|c|c|c|}
\hline$T_{7}$ & $T_{8}$ & $T_{9}$ & $T_{10}$ & $T_{11}$ & $T_{12}$ & $T_{13}$ \\
\hline 0.8032 & 0.7863 & 0.7695 & 0.7527 & 0.7361 & 0.7196 & 0.7031 \\
\hline
\end{tabular}

Discount Factors from $T_{14}$ to $T_{20}$

\begin{tabular}{|c|c|c|c|c|c|c|}
\hline$T_{14}$ & $T_{15}$ & $T_{16}$ & $T_{17}$ & $T_{18}$ & $T_{19}$ & $T_{20}$ \\
\hline 0.6868 & 0.6707 & 0.6546 & 0.6387 & 0.6231 & 0.6080 & 0.5932 \\
\hline
\end{tabular}

Table 1: Discount factors in the initial discount curve.

There exist other more general yield curve models, like the non-parallel shifts model (Hagan, 2003). The calculations on other models can be performed in an almost identical manner. We expect that the illustrated features would be very similar.

\section{CMS caplets}

Based on the replication formula (2.6), we find the replicating portfolios of a set of at-the-money CMS caplets using payer swaptions of varying discrete strikes. The respective strike rate $K$ of the at-the-money CMS calpets, presented in Table 2 , is chosen to be the prevailing forward swap rates which are given by the following equation:

$$
S\left(0 ; T_{0}, T_{n}\right)=\frac{B\left(0, T_{0}\right)-B\left(0, T_{n}\right)}{\sum_{k=1}^{n} \delta_{k} B\left(0, T_{k}\right)} .
$$

The subinterval of the strike price $\Delta x$ is set to be $1 \%$. The upper bound on the strikes of the these swaptions is set to be $20 \%$. The notional of each of these swaptions can be computed using Eq. (2.5). 


\begin{tabular}{c|ccc}
$n$ & 8 & 10 & 20 \\
\hline$K$ & $4.89 \%$ & $4.80 \%$ & $4.81 \%$
\end{tabular}

Table 2: Values of the strike rate under various tenor structures.

In Figures 2(a) and 2(b), we show the plot of the notional values of the payer swaptions with varying strikes corresponding to different tenors, where $n=8,10,20$, based on the standard model and parallel shifts model, respectively. The swaption with strike $K$ (the same strike of the CMS caplet) is seen to be the dominant one (in terms of notional and actual dollar value) in the replicating portfolio. The convexity correction is provided by the other payer swaptions at higher strikes. As revealed in Figures 2(a) and 2(b), the notional amounts of these swaptions are relatively small compared to that of the dominant swaption at strike $K$. Since $G\left(S_{T_{0}}\right)$ is almost linear in $S_{T_{0}}$, the notional values of the swaptions relevant for the convexity correction are almost equal in value. When the tenor of the underlying CMS rate is lengthened, corresponding to an increase in value of $n$, the convexity correction becomes more significant. Thus the difference between the notional of the dominant swaption at strike $K$ and that of any swaption at a higher strike decreases. This phenomenon is confirmed by the plots shown in Figures 2(a) and 2(b).

To illustrate the impact on the notional values in the replicating portfolio with regard to the choice of the yield curve model, we compute the numerical values of the notional of the payer swaptions against varying strike rate under the two yield curve models with different tenor structures (varying number of payment dates $n$ ). The results are presented in Table 3 .

\begin{tabular}{|c|c|c|c|c|c|c|c|c|}
\hline \multicolumn{3}{|c|}{$n=8$} & \multicolumn{3}{|c|}{$n=10$} & \multicolumn{3}{|c|}{$n=20$} \\
\hline \multirow[b]{2}{*}{$\begin{array}{l}\text { Strike } \\
\text { rate }\end{array}$} & \multicolumn{2}{|c|}{ Notional } & \multirow[b]{2}{*}{$\begin{array}{c}\text { Strike } \\
\text { rate }\end{array}$} & \multicolumn{2}{|c|}{ Notional } & \multirow[b]{2}{*}{$\begin{array}{l}\text { Strike } \\
\text { rate }\end{array}$} & \multicolumn{2}{|c|}{ Notional } \\
\hline & $\begin{array}{c}\text { Standard } \\
\text { model }\end{array}$ & $\begin{array}{c}\text { Parallel } \\
\text { shifts } \\
\text { model }\end{array}$ & & $\begin{array}{c}\text { Standard } \\
\text { model }\end{array}$ & $\begin{array}{c}\text { Parallel } \\
\text { shifts } \\
\text { model }\end{array}$ & & $\begin{array}{c}\text { Standard } \\
\text { model }\end{array}$ & $\begin{array}{c}\text { Parallel } \\
\text { shifts } \\
\text { model }\end{array}$ \\
\hline 0.0489 & 0.2802 & 0.2873 & 0.0480 & 0.2314 & 0.2375 & 0.0481 & 0.1347 & 0.1380 \\
\hline 0.0589 & 0.0090 & 0.0085 & 0.0580 & 0.0094 & 0.0091 & 0.0581 & 0.0109 & 0.0109 \\
\hline 0.0689 & 0.0090 & 0.0081 & 0.0680 & 0.0095 & 0.0088 & 0.0681 & 0.0111 & 0.0110 \\
\hline 0.0789 & 0.0091 & 0.0079 & 0.0780 & 0.0096 & 0.0086 & 0.0781 & 0.0114 & 0.0111 \\
\hline 0.0889 & 0.0091 & 0.0077 & 0.0880 & 0.0097 & 0.0085 & 0.0881 & 0.0116 & 0.0113 \\
\hline 0.0989 & 0.0092 & 0.0075 & 0.0980 & 0.0097 & 0.0084 & 0.0981 & 0.0119 & 0.0114 \\
\hline
\end{tabular}

Table 3: Notional values of the payer swaptions in the replication portfolio of the CMS caplet under the standard model and the parallel shifts model.

\section{Annuity options}

We apply the replication formula (3.8) to find the notional values of the receiver swaptions in the replication of an annuity option. We assume a uniform annuity rate of $\$ 1$ in our calculations. The strike price $A$ of the annuity option is set equal to the forward price of the annuity such that the annuity option is initiated at-the-money.

In a similar manner, we plot the notional values of the receiver swaptions in the replicating portfolio corresponding to different tenors in Figures 3(a) and $3(\mathrm{~b})$. Since $G(x)$ is "almost" linear in both the standard model and parallel 
shifts model and so $G^{\prime \prime}(x) \approx 0$, the notional values of all receiver swaptions other than the dominant swaption are very small. If we neglect the "small" convexity of the function $G(x)$, the annuity option is almost like a receiver swaption with notional $\left(A-c_{0}\right) G^{\prime}(K)$ and strike rate $K$, where $K$ is the unique root of $\widetilde{f}(x)=$ $\mu-\left(A-c_{0}\right) G(x)$. The values of $K$ under various tenor structures are summarized in Table 4.

\begin{tabular}{c|ccc} 
Number of payment dates, $n$ & 8 & 10 & 20 \\
\hline Standard model & $4.89 \%$ & $4.80 \%$ & $4.81 \%$ \\
Parallel shifts model & $4.89 \%$ & $4.80 \%$ & $4.81 \%$
\end{tabular}

Table 4: Values of the strike rate $K$ under various tenor structures and yield curve models.

Due to the small convexity in $G(x)$, one would short a small number of units of receiver swaptions at strikes lower than $K$ in the replicating portfolio [see Figures 3(a) and 3(b)].

\section{Conclusion}

We have proposed a generalized static replication formula by exploring the linkage between replication, convexity correction and numeraire change. Our static replication approach is under the one factor framework, where all uncertainty in the term structure of the yield curve is captured by one single variable, say the swap rate. The generalized replication formula allows the adoption of wider choices of option-type products in the replicating portfolio and the use of numeraires that are preferred for affective valuation of a contingent claim. We demonstrate the use of the generalized replication approach for hedging exotic swap products and annuity options using various types of swaptions. Interestingly, the convexity of the functional dependence of bond-annuity ratio on swap rate enters into the replication formulas of these exotic interest rate instruments. The degree of the convexity determines the notional amounts of the swaptions in the replicating portfolio, thus illustrating the interplay between convexity and replication.

\section{References}

[1] Carr, P. and D. Madan, 1998. Towards a theory of volatility trading; in R.A. Jarrow, ed.: Volatility: New estimation Techniques for Pricing Derivatives (RISK Publications, London).

[2] Hagan, P., 2003. Convexity conundrums: Pricing CMS swaps, caps, and floors. Wilmott Magazine, March, p.38-44.

[3] Mercurio, F. and A. Pallavicini, 2006. Smiling at convexity: bridging swaption skews and CMS adjustments, working paper of Banca IMI.

[4] Pelsser, A., 2003a. Mathematical foundation of convexity correction. Quantitative Finance, 3, p.59-65. 
[5] Pelsser, A., 2003b. Pricing and hedging guaranteed options via static option replication. Insurance: Mathematics and Economics, 33, p.283-296.

[6] Reiner, E. and M. Sellami, 2006. Valuation approaches to constant maturity swap (CMS) options. Investment Acumen: AXA Investment Managers' Research Review, p.36-41.

\section{Appendix A Proof of Proposition 1}

First, we recall the martingale pricing formulas:

$$
\begin{aligned}
V_{0} & =M(0) E_{\mathbb{Q}_{M}}\left[f\left(X_{T}\right)\right]=N(0) E_{\mathbb{Q}_{N}}\left[f\left(X_{T}\right) \frac{M(T)}{N(T)}\right] \\
& =N(0) E_{\mathbb{Q}_{N}}\left[f\left(X_{T}\right) \Lambda\left(X_{T}\right)\right],
\end{aligned}
$$

where $\mathbb{Q}_{M}$ and $\mathbb{Q}_{N}$ are the equivalent martingale measures associated with the numeraires $M(t)$ and $N(t)$, respectively. Suppose we choose $\kappa \geq 0$ such that $f(\kappa)=0$. Thus, $w(\kappa)=\frac{f(\kappa) \Lambda(\kappa)}{g(\kappa)}=0$. We apply the Carr-Madan formula (3.1) to the payoff function $w\left(X_{T}\right)$ and obtain

$$
\begin{aligned}
w\left(X_{T}\right)= & w^{\prime}(\kappa)\left[\left(X_{T}-\kappa\right)^{+}-\left(\kappa-X_{T}\right)^{+}\right] \\
& +\int_{0}^{\kappa} w^{\prime \prime}(x)\left(x-X_{T}\right)^{+} d x+\int_{\kappa}^{\infty} w^{\prime \prime}(x)\left(X_{T}-x\right)^{+} d x
\end{aligned}
$$

By multiplying both sides of the above equation by $g\left(X_{T}\right)$ and taking expectation under the martingale measure $\mathbb{Q}_{N}$, we obtain

$$
\begin{aligned}
E_{\mathbb{Q}_{N}}\left[f\left(X_{T}\right) \Lambda\left(X_{T}\right)\right]= & w^{\prime}(\kappa) E_{\mathbb{Q}_{N}}\left[g\left(X_{T}\right)\left(\left(X_{T}-\kappa\right)^{+}-\left(x-X_{T}\right)^{+}\right)\right] \\
& +\int_{0}^{\kappa} w^{\prime \prime}(x) E_{\mathbb{Q}_{N}}\left[g\left(X_{T}\right)\left(x-X_{T}\right)\right]^{+} d x \\
& +\int_{\kappa}^{\infty} w^{\prime \prime}(x) E_{\mathbb{Q}_{N}}\left[g\left(X_{T}\right)\left(X_{T}-x\right)\right]^{+} d x .
\end{aligned}
$$

The values of the call-type and put-type replicating instruments are given by

$$
C_{0}(x)=N(0) E_{\mathbb{Q}_{N}}\left[g\left(X_{T}\right)\left(X_{T}-x\right)^{+}\right]
$$

and

$$
P_{0}(x)=N(0) E_{\mathbb{Q}_{N}}\left[g\left(X_{T}\right)\left(x-X_{T}\right)^{+}\right],
$$

respectively; so we finally obtain

$$
\begin{aligned}
V_{0}= & w^{\prime}(\kappa)\left[C_{0}(\kappa)-P_{0}(\kappa)\right] \\
& +\int_{0}^{\kappa} w^{\prime \prime}(x) P_{0}(x) d x+\int_{\kappa}^{\infty} w^{\prime \prime}(x) C_{0}(x) d x .
\end{aligned}
$$


(a) Standard model

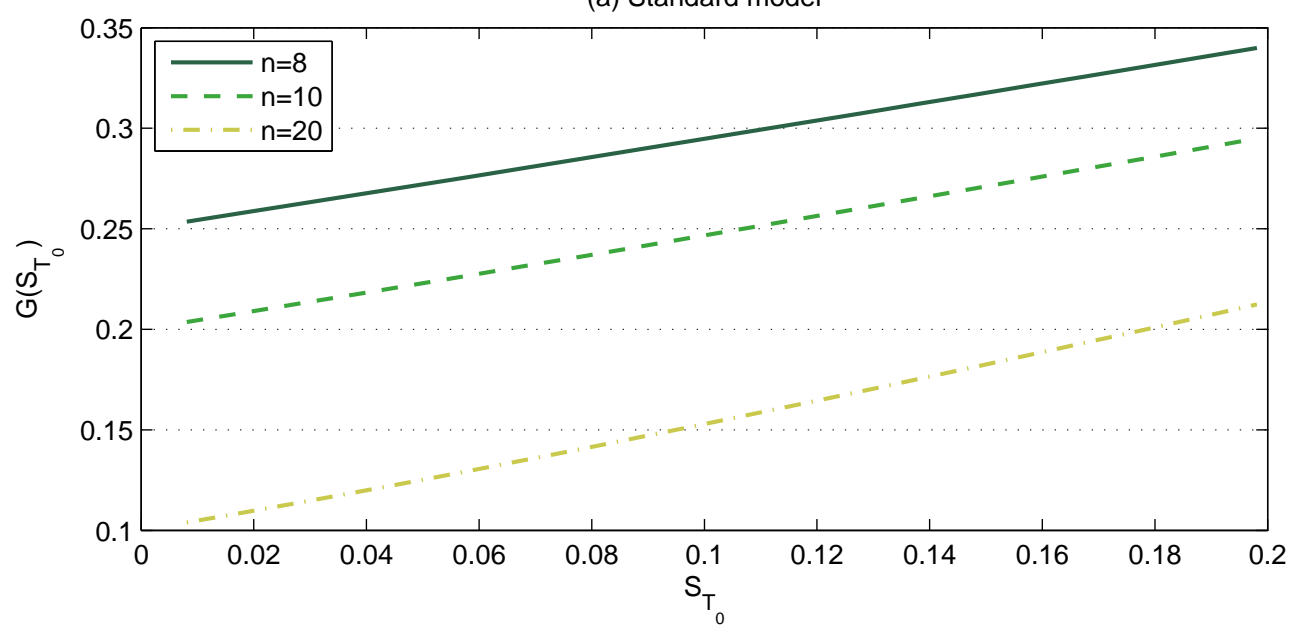

(b) Parallel shifts model

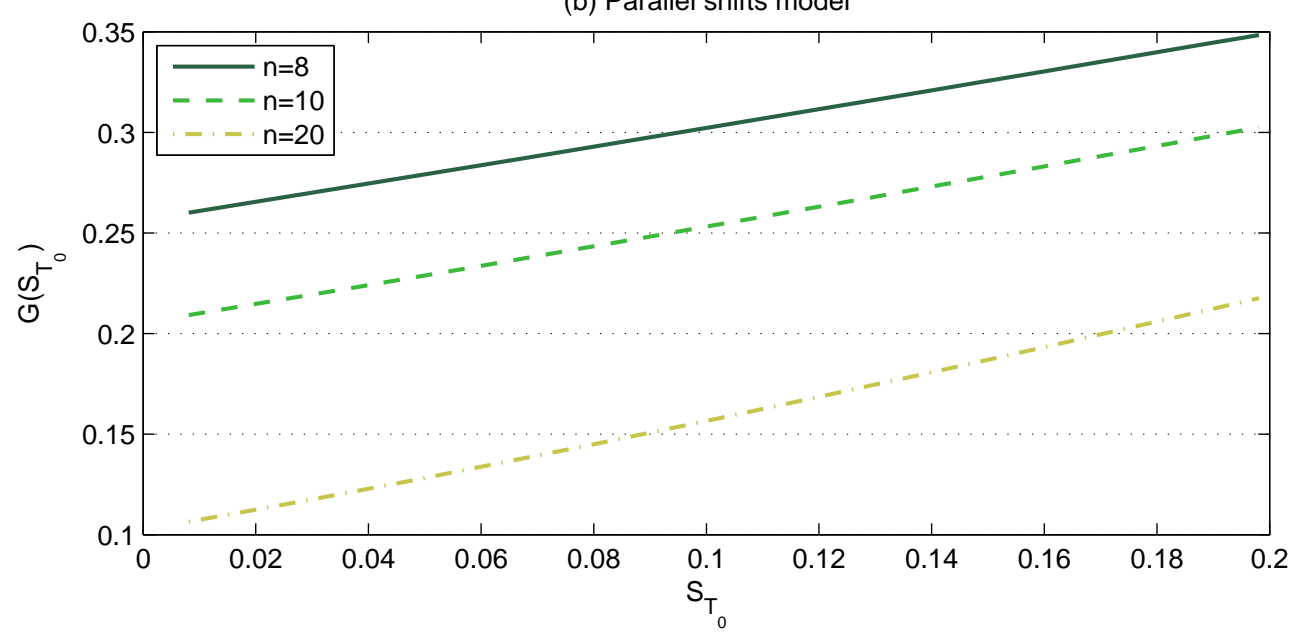

Figure 1: Plot of $G\left(S_{T_{0}}\right)$ against $S_{T_{0}}$ under the assumption of (a) standard model, (b) parallel shifts model. The "almost linear" property of $G$ is observed. 
(a) Standard model

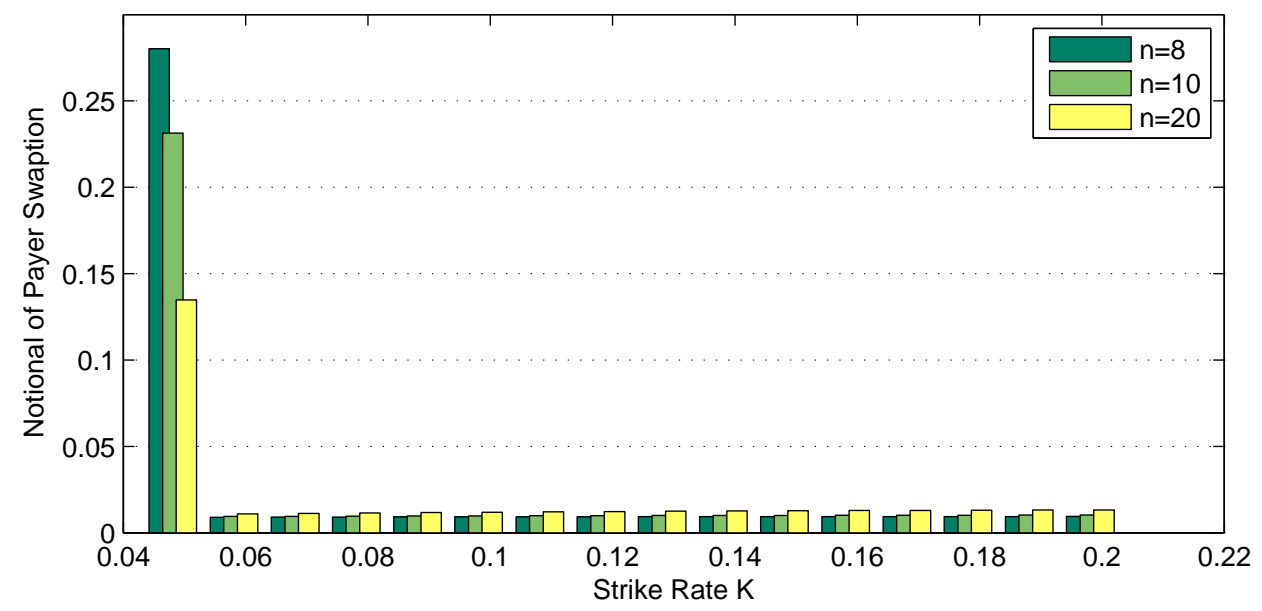

(b) Parallel shifts model

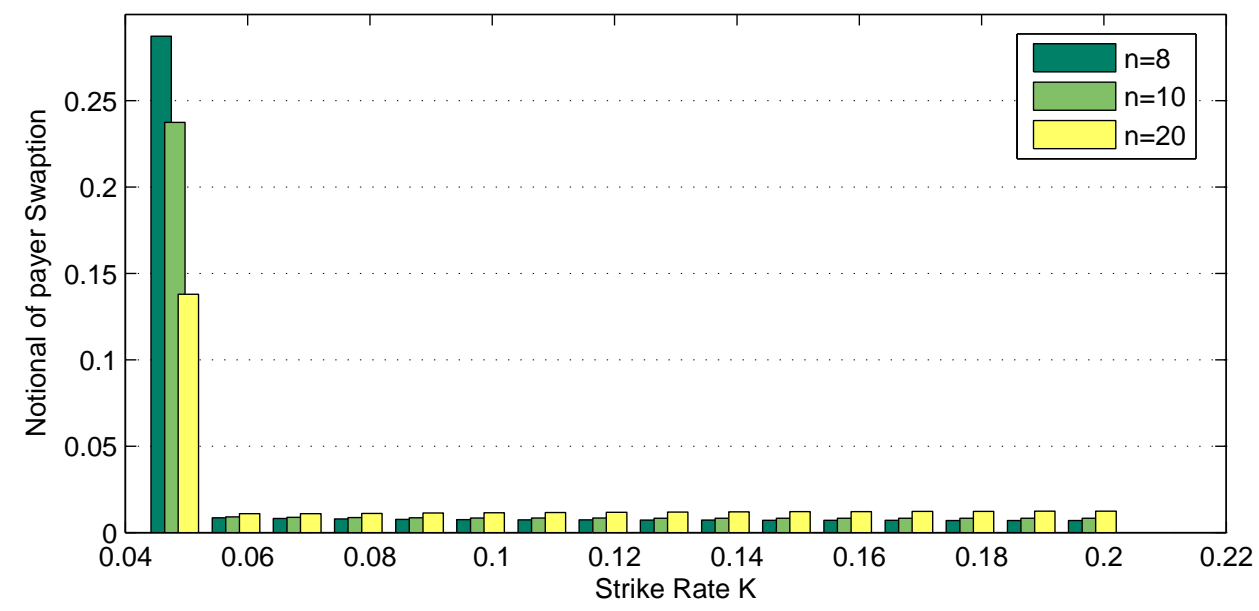

Figure 2: Plot of the notional of the payer swaption against strike rate under the assumption of (a) standard model, (b) parallel shifts model, and corresponding to different tenor structure (signified by the number of payment dates $n$ in the tenor). The payer swaption with the same strike as that of the CMS caplet is the dominant one. 
(a) Standard model

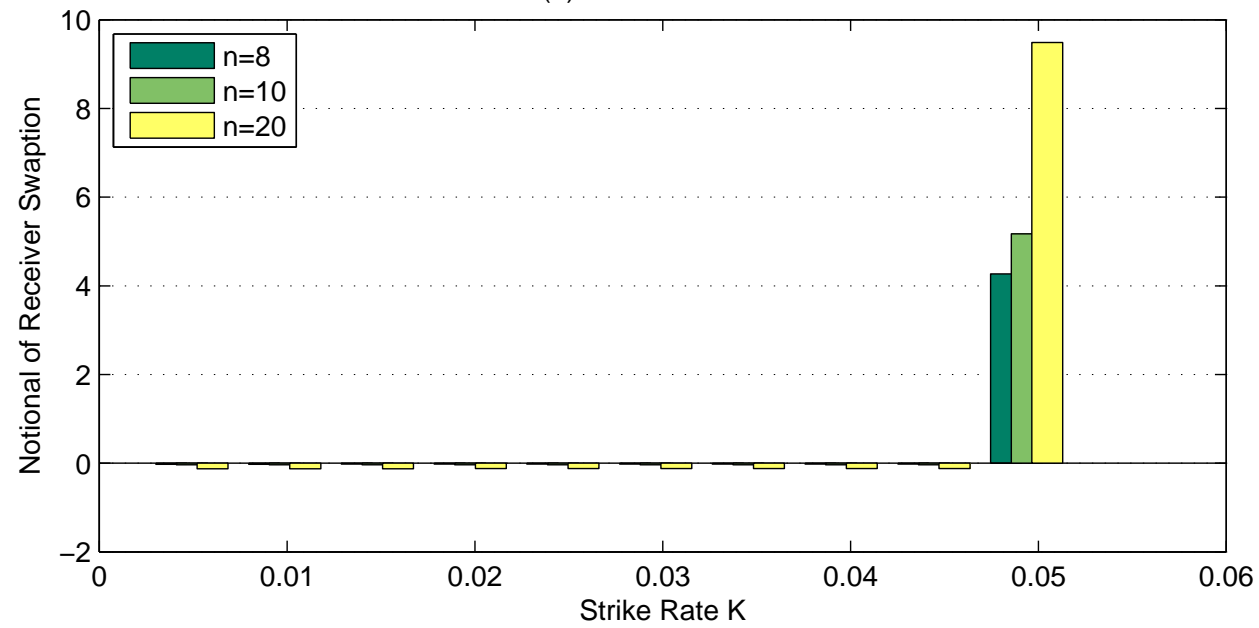

(b) Parallel shifts model

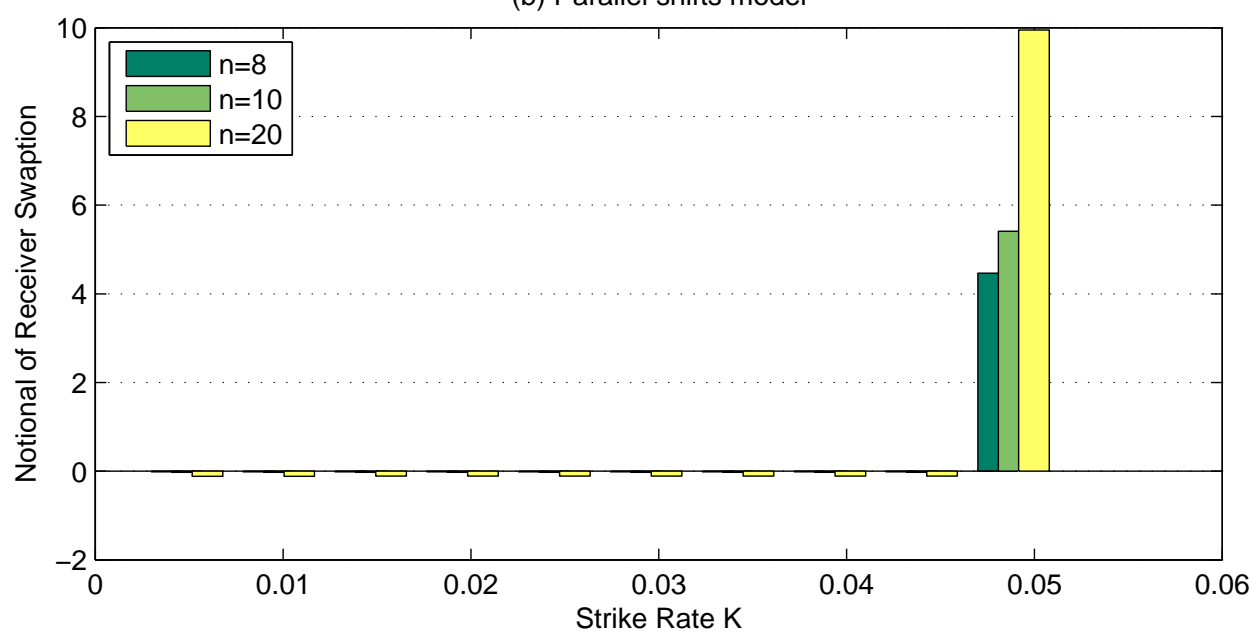

Figure 3: Plot of the notional of the receiver swaption against strike rate under the assumption of (a) standard model, (b) parallel shifts model, and with varying tenor structure (signified by the number of payment dates $n$ in the tenor). The notional amounts of the receiver swaptions relevant for convexity correction are seen to be very small due to the "almost linear" property of $G$. 